\title{
Primitive pleural lymphoma developed on sequelae of pneumothorax therapeutics
}

\section{Lymphome pleural primitif développé sur des séquelles de pneumo- thorax thérapeutique}

\author{
1: Faculté des Sciences et Techniques de la Santé. Université de Conakry \\ 2: Faculté des Sciences de la Santé. Université de Lomé \\ 3: Service de Pathologies Respiratoires CHU Limoges. Hôpital du Cluzeau
}

BD. Diallo ${ }^{1}$, OH. Diallo', STA. Adamabounou ${ }^{2}$, D. Toure ${ }^{1}$, MH. Camara ${ }^{1}$, B. Melloni ${ }^{3}$

\section{ABSTRACT}

We report the medical observation of an 84-year-old patient with pleural lymphoma B developed on sequelae of pneumothorax therapeutic tuberculosis in 1949.

This is a case of large EBV +, CD20 +, CD $79 \mathrm{~A}+, \mathrm{CD} 2+, \mathrm{CD} 3+$ lymphoma B (aberrant T markers).

The patient was treated with chemotherapy which resulted in his death, 7 months after the start of treatment.

Pneumothorax-associated lymphoma is a rare condition and will disappear with the aging of the last patients treated with this technique.

KEYWORDS: Pleural lymphoma; Pneumothorax; Tuberculosis.

\section{RÉSUMÉ}

Nous rapportons l'observation médicale d'un patient de 84 ans, porteur d'un lymphome pleural B développé sur des séquelles de pneumothorax thérapeutique d'une tuberculose en 1949.

Il s'agit d'un cas de lymphome B à grandes cellules EBV+, CD20 +, CD 79 A +, CD2 ${ }^{+}, \mathrm{CD}^{+}$(marqueurs T aberrants).

Le patient avait pris en charge avec une chimiothérapie qui s'est soldée par son décès, 7 mois après le début de la prise en charge.

Le lymphome associé au Pneumothorax thérapeutique est une affection rare et disparaitra avec le vieillissement des derniers patients ayant été traité par cette technique.

MOTS CLÉS: Lymphome pleural; Pneumothorax; Tuberculose. 


\section{INTRODUCTION}

Avant l'ère des antibiotiques antituberculeux, plusieurs techniques thérapeutiques ont été utilsés. L'un des premiers traitements était la collapsothérapie proposée par Forlanini dès 1882 [1]. Le principe consistait à créer un pneumothorax dans le but d'affaisser le poumon et donc de priver le BK d'oxygène, ce qui permettait d'assurer la guérison à certains malades au prix d'importantes séquelles [1].

Le lymphome pleural associé au pyothorax représente une complication tardive d'un empyème ou $d^{\prime}$ un pneumothorax iatrogène pour traitement $d$ 'une tuberculose pulmonaire. C'est ainsi que le terme de "pyothorax associated lymphoma" (PAL) a été proposé dans la littérature en 1987 par Luchi [2]. Il s'agit d'une entité anatomo-clinique parfaitement reconnue même si cette pathologie reste rare [3]. Le type histologique est le plus souvent un lymphome de type B à grandes cellules, même si la plupart des types histologiques peuvent être observés [4].Le PAL est essentiellement décrit au Japon.. En Afrique, cette entité reste méconnue, nous n'avons trouvé aucun cas décrit dans la littérature. Cette affection peut survenir en moyenne 30 à 40 ans après le pneumothorax thérapeutique $[4,5]$. Le rôle étiologique du virus de l'Epstein Barr(EBV) reste très probable bien que les causes environnementales ne soient pas exclues [6] . Le pronostic reste sombre, la survie à 5ans est de l'ordre de $0,35 \%$ quel que soit le traitement institué $[3,4]$.

Le but de notre travail est de rapporter l'observation médicale d'un patient hospitalisé pour masse pulmonaire droite à investiguer dans le service de Pathologies Respiratoires du CHU de Limoges (Hôpital du Cluzeau).

\section{OBSERVATION MEDICALE}

Monsieur B.P., âgé 84 ans, ancien cadre de banques, habitant Limoges a été admis au service de Pathologies Respiratoire du CHU de Limoges pour bilan d'une masse pulmonaire dans un contexte d'altération de l'état général, il avait comme antécédents une tuberculose pulmonaire bilatérale traitée par collapsothérapie en 1949 ; Un cancer colorectal opéré en 1990 ; une Cholécystectomie ; une HTA; un infarctus du myocarde en 1992 ; une mise en place d'un pacemaker ; un pontage aorto-coronarien ; un syndrome de Guillain Barré en 2004 ; un œsophagite stade I, une hernie hiatale; un nodule thyroïdien du lobe gauche.

Mr B.P. a été adressé au service de pathologie respiratoire pour bilan d'une masse pulmonaire dans un contexte d'altération de l'état général associé à un syndrome inflammatoire biologique.
Le patient était conscient, bien orienté, apyrétique. L'état général était conservé (PS=1). Il présentait une anorexie modérée. Il pesait $75 \mathrm{~kg}$ pour $1,73 \mathrm{~m}$, soit un IMC de 25,06.

Au niveau pulmonaire, on notait une abolition du murmure vésiculaire au niveau de l'hémi champ supérieur droit avec une matité à la percussion. L'auscultation pulmonaire gauche était normale. La radiographie du thorax avait permis d'objectiver une masse homogène dense occupant le tiers supérieur du champ pulmonaire droit (Figure1).

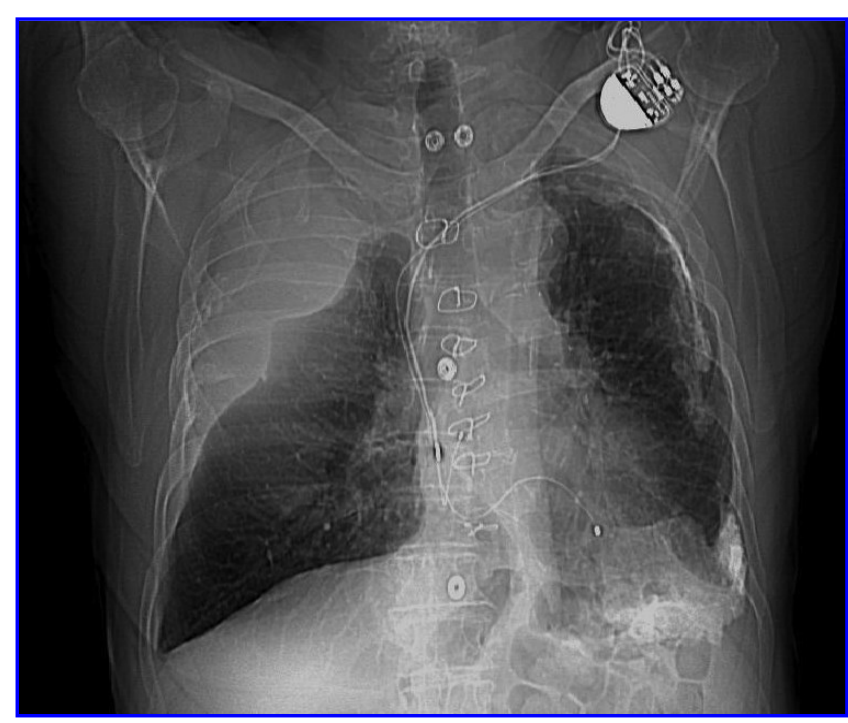

FIGURE 1. Radiographie standard du thorax (incidence de face).

Le Scanner thoraco-abdomino-pelvien visualisait une masse tumorale pariétale thoracique droite avec un envahissement parenchymateux pulmonaire, osseux et des parties molles ainsi que des adénopathies médiastinales supérieures (Figure 2)

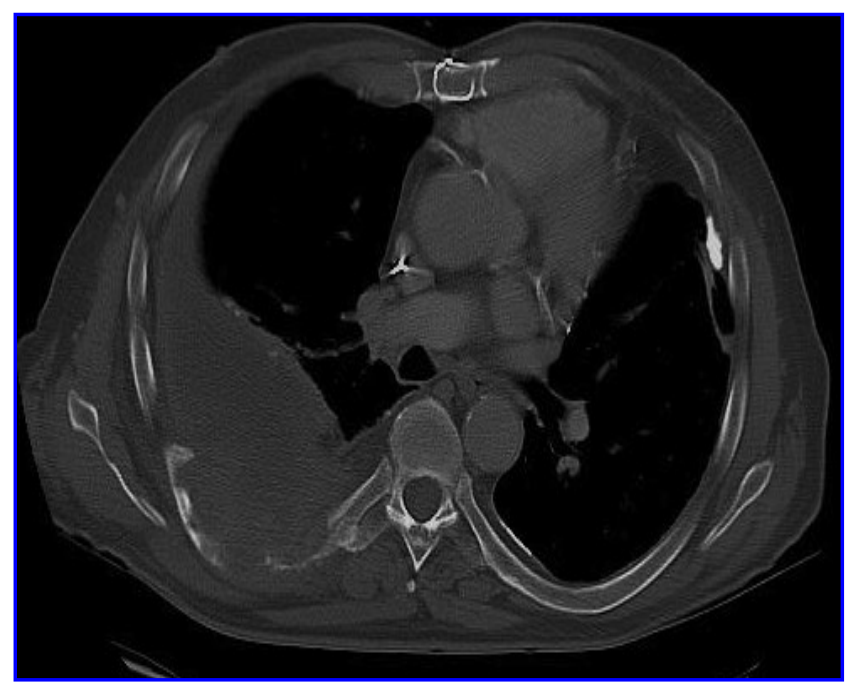

FIGURE 2. Coupe médiastinale du TDM thoracique de Monsieur BP. 
La Tomographie à émission de positons (TEP) retrouvait une lésion hyper métabolique de la paroi avec envahissement osseux (SUVmax=18) (Figure 3).

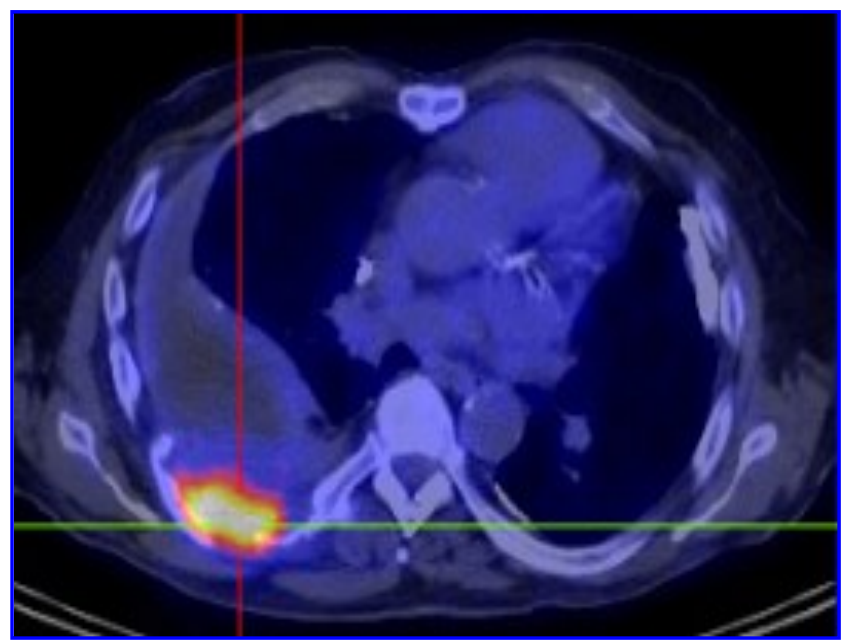

FIGURE 3. Tomographie à émission de positons (TEP) retrouvait une lésion hyper métabolique de la paroi avec envahissement osseux (SUVmax $=18)$.

L'examen anatomo-pathologique avait conclu à un lymphome B à grandes cellules EBV+ développées sur séquelles pleurales de pneumothorax thérapeutique, $\mathrm{CD} 2 \mathrm{O}^{+}, \mathrm{CD} 79 \mathrm{~A}^{+}, \mathrm{CD}^{+}, \mathrm{CD}^{+}$(marqueurs T aberrants).

L'électrophorèse des protéines montrait une hypo albuminémie à 31,1 g/l. Le patient a été transféré en service d'hématologie pour prise en charge. Une chimiothérapie a été instituée à base de ZEM (hydrarubicyne ciclophosphamide, méthylpridnisolone). Apres quatre cures, l'évaluation avait conclu à l'absence de réponse thérapeutique. Une deuxième ligne par ETOPOSIDE/OLOXANE a donc été instituée. Après la deuxième cure, une nouvelle étude conclut à une progression de la maladie. En RCP, la décision d'arrêter la chimiothérapie a été prise. Le patient a alors été mis en soins palliatifs.

Il est décédé sept mois après le diagnostic

\section{DISCUSSION}

Cette observation évoque un cas de lymphome $B$ à grandes cellules, EBV positif chez un homme de 84 ans. Il avait présenté une tuberculose pulmonaire traitée par une collapsothérapie, il y'a 68 ans.. Le PAL est une affection rare comme en témoigne les différents auteurs [3,4]. En France, une vingtaine de cas seulement avaient été rapportés dans la littérature jusqu'en 2007. Le PAL est une affection décrite essentiellement au Japon. Cela serait dû au fait la collapsothérapie y a été largement pratiquée dans les années 1930 - 40. Cependant un facteur environnemental n'est pas exclu. En Afrique, aucun cas n'a été décrit, cela serait au dû au fait que cette thérapeutique n'a probablement pas été utilisée sur le continent dans la prise en charge thérapeutique de la tuberculose.

Le délai moyen de survenue d'un PAL était en moyenne de 43 ans avec des extrêmes de 19 et 64 ans au Japon [4] dans la série de H. Narimatsu et al. Il était de 54 et 55 ans selon P. Saint-Blanchard en 2007 [5] sur une publication de deux cas en France. Ce délai était plus long dans notre cas (69 ans).

Cette affection s'observe en règle chez des sujets âgés, à un âge moyen compris entre 65 et 70 ans. Dans l'étude de Lavole et al. [3], l'âge des patients était respectivement de 66 et de 81 ans en 2006 en France. Notre patient avait un âge compris dans cette tranche d'âge. Narimatsu H. et al. avaient trouvé un âge médian de 70 ans sur un effectif de 98 patients [4]. Cet âge avancé s'explique par le long délai de survenue du processus néoplasique sur les séquelles de pneumothorax. La cause des PAL demeure méconnue et le rôle spécifique de Mycobacterium tuberculosis peut être exclu, de même qu'un contexte d'immunodépression [5]. En revanche, l'interleukine 6 et l'EBV semblent être des facteurs d'oncogenèse.

Une étude comparant 34 lymphomes sur pyothorax et 16 pyothorax chroniques sans lymphome a permis de mettre en évidence la présence d'EBV dans les 34 lymphomes alors qu'un seul pyothorax chronique sans lymphome exprimait l'EBV [3].

Notre patient n'avait présenté aucune maladie liée à une infection virale par EBV.

Du point de vue physiopathologique, l'inflammation pleurale chronique pourrait favoriser la prolifération des lymphomes B infectés par EBV leur permettant d'échapper à la surveillance immunitaire soit par blocage mécanique par la coque pleurale des lymphocytes $\mathrm{T}$ ou par la production locale de cytokine (IL-6). Dès 1993, la présence du génome de l'EBV (latent membran protéin) et d'EBV -associated nuclear antigen (EBNA-2) dans les lymphomes associés au pyothorax a été décrite [4]. Dans la majorité des cas, il s'agit de lymphomes à grandes cellules de phénotype $\mathrm{B}$. Notre patient répond à ce type histologique. Sur le plan évolutif, les moyennes de survie rapportées sont de 3 à 6 mois quel que soit le traitement effectué: chimiothérapie, association radiochimiothérapie, soins palliatifs. La chimiothérapie offre des résultats inconstants et toujours transitoires $[4,5]$. Notre malade, après deux lignes de chimiothérapie était en progression et il est décédé en soins palliatifs sept mois de prise en charge. La survie à 5 ans est exceptionnelle. L'âge élevé au moment du 
diagnostic et l'altération de l'état général semblent être des éléments de mauvais pronostic [4].

\section{CONCLUSION}

Le lymphome pleural primitif sur séquelles de pneumothorax thérapeutique est une affection rare et de

\section{CONFLIT D'INTÉRÊTS}

Aucun.

\section{RÉFERENCES}

1. D. Fichet, S. El Mechaal, Historique du traitement de la tuberculose de 1882 à 1965.

2. EMC Pneumologie 2007 Pages 19-36.

3. Keiji Iuchi Katsuyuki, Aozasa Satoru Yamamoto, Takashi Mori, Kazuo Tajima Keisuke, Minato Kiyoshi Mukai Hikotaro, Komatsu Toshiyuki Tagaki Yoichiro Kobashi; Non-Hodgkin's Lymphoma of the Pleural Cavity Developing from Long-standing Pyothorax. Summary of Clinical and Pathological Findings in Thirty-seven Cases Jpn. J. Clin. Oncol, 1989 ; 19 : 249257. mauvais pronostic. Nous avons rapporté ici l'observation d'un patient de 78 ans, chez qui le diagnostic a été posé après une biopsie chirurgicale. L'association au virus de l'Epstein Barr est quasi constante.

Notre observation fera partie fort probablement des derniers cas décris dans la littérature.
4. Narimatsu H, Ota Y, Kami M, Takeuchi K, Suzuki R, Matsuo K, et al. Clinicopathological features of pyothorax-associated lymphoma; a retrospective survey involving 98 patients. Ann Oncol. 2007 18(1):122-8.

5. P.Saint-Blancard A, Harket G, Defuentes A, -L.de Fonclare A, Bonnichon T, de Revel O, Berets J.Margery Lymphome pleural primitif: une complication tardive de la collapsothérapie chez des anciens tuberculeux: À propos de deux nouveaux cas Rev aMal. Respir ; 2001 $18: 72-74$ 